\title{
Téoros
}

Revue de recherche en tourisme

\section{Contribution de la formation au développement du tourisme} au Maroc

\section{Fatima Zohra Guertaoui}

Volume 24, numéro 1, printemps 2005

Maroc, réalités et défis

URI : https://id.erudit.org/iderudit/1071105ar

DOI : https://doi.org/10.7202/1071105ar

Aller au sommaire du numéro

Éditeur(s)

Université du Québec à Montréal

ISSN

0712-8657 (imprimé)

1923-2705 (numérique)

Découvrir la revue

Citer cet article

Guertaoui, F. Z. (2005). Contribution de la formation au développement du tourisme au Maroc. Téoros, 24(1), 20-24. https://doi.org/10.7202/1071105ar d'utilisation que vous pouvez consulter en ligne.

https://apropos.erudit.org/fr/usagers/politique-dutilisation/ 


\section{Contribution de la formation au développement du tourisme au Maroc}

\begin{abstract}
Fatima Zohra Guertaoui
Le tourisme international a progressé en moyenne de $7 \%$ par an depuis 1950. Aux dires des experts, il continuera sur sa lancée à des rythmes satisfaisants $(4,5 \%$ au cours des vingt prochaines années), malgré la persistance d'un climat mondial peu propice aux voyages (guerres, attentats, épidémie). Cette progression rassure et surprend à la fois. Déjà, au cours de ces dernières années, la croissance du tourisme international a été plus rapide que celle de l'économie mondiale dans son ensemble. Avec quelque 715 millions d'arrivées en 2000, le tourisme mondial semble défier les difficultés de l'heure et se présente comme une des plus résistantes branches d'activités de la planète aux différentes crises (OMT, 2001).
\end{abstract}

Après avoir bénéficié d'une augmentation quantitative, la demande touristique met le cap sur la qualité. Celle-ci a le mérite d'agir en amont (émission) et en aval (réception). De ce fait, les voyages qui participent incontestablement au remodelage du style de vie des pays développés ont aussi, sur ceux dits en développement, des impacts variés. Et si les classes aisées étaient les plus concernées, le personnel qui travaillent dans l'industrie serait le bénéficiaire de ce saut qualitatif (formation et revenu).

Désormais, les voyages sont plus courts, mais plus fréquents et mieux ciblés, ce qui permet une bonne répartition, dans le temps et dans l'espace, des départs et des séjours. Des projets innovants voient le jour dans le but de diversifier l'offre touristique. Ainsi est née la notion de "PAYS » qui permet de structurer et de valoriser une offre de tourisme rural « labellisé » qui met en valeur les riches caractéristiques du patrimoine na-

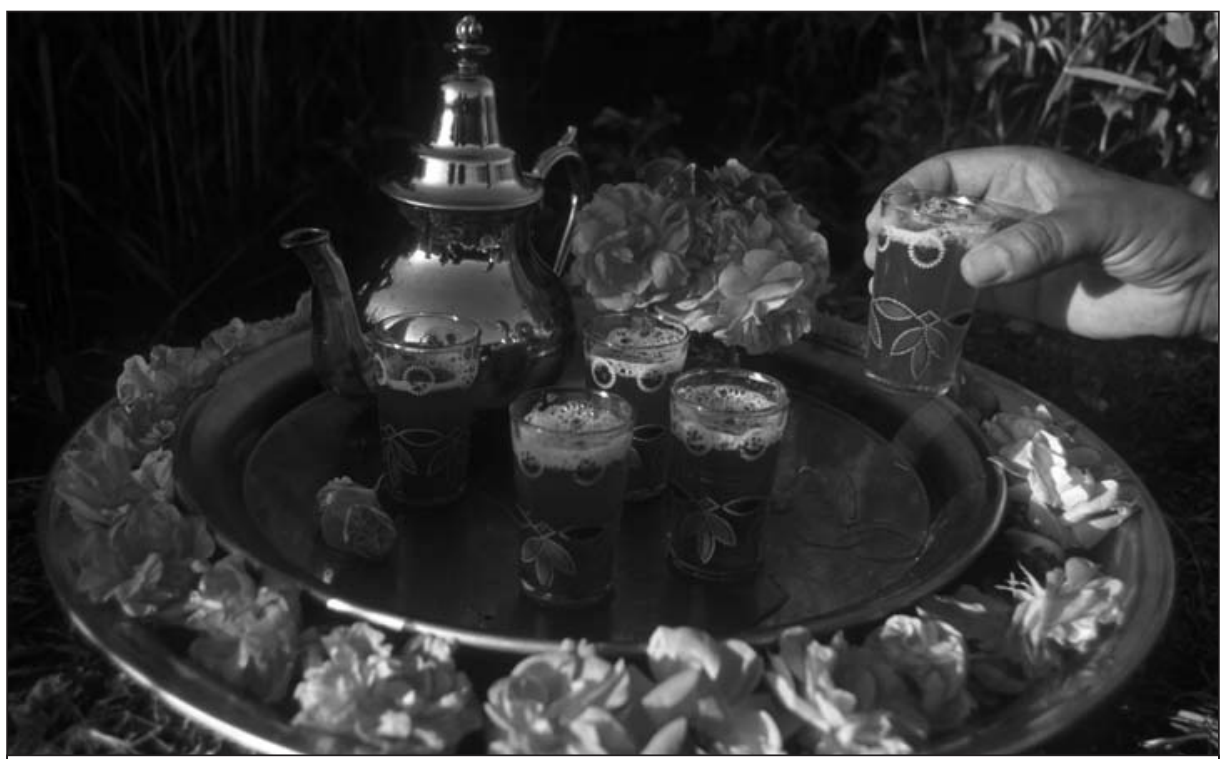

Cérémonie du thé, vallée du Dads (Maroc).

Photo : tourisme-marocain.com

turel et culturel marocain. Aujourd'hui, il y a plusieurs «pays d'accueil touristiques » qui quadrillent le territoire marocain. Ils devraient permettre de "mettre en marché » des itinéraires touristiques de randonnées ainsi qu'une offre d'hébergement sous forme de gîtes ruraux dont les populations locales bénéficieront largement.

Dans les différentes branches du tourisme classique, l'heure est à la qualité des produits et services. Et c'est ainsi que les touristes sont correctement accueillis, mieux informés et mieux servis. On sait qu'aujourd'hui le touriste est de plus en plus sensible à la qualité des prestations et à la protection de l'environnement, dimensions que le tourisme favorise et que les professionnels du tourisme savent prendre en compte.

Parallèlement à cette évolution qualitative, la diffusion de nouvelles techniques de l'informa- tion permet aux professionnels du tourisme de proposer plus efficacement leurs produits et services à des clientèles diversifiées et exigeantes, qui n'ont que l'embarras du choix face à la multitude des destinations proposées.

Des besoins accrus en personnel qualifié, aux compétences très pointues, sont vivement ressentis au sein de la profession pour être à même de répondre aux demandes d'une clientèle de plus en plus diversifiée et exigeante. Un personnel spécialisé et hautement qualifié en gestion des ressources humaines est de ce fait particulièrement important dans ce contexte d'évolution des métiers du tourisme. La concurrence aidant, les cadres de direction des entreprises touristiques s'ingénient à exceller dans les stratégies de gestion, de planification et de marketing afin d'être en mesure d'identifier, de programmer et d'exploiter précocement les tendances du marché. 
Par ailleurs, la sensibilisation aux questions de santé et de sécurité, l'assimilation de la «culture d'entreprise » au sein de l'établissement, la capacité d'apporter à la clientèle de l'information de plus en plus précise et efficace, sont des aptitudes essentielles pour les employés de l'industrie touristique. D'autres qualités encore relèvent de compétences distinctives: des connaissances linguistiques et de la sensibilité aux différences interculturelles sont recherchées.

En outre, les salariés du secteur sont appelés à renforcer leurs aptitudes sur le plan social et humain, afin de jouer pleinement leur rôle dans le cadre de l'esprit et du travail d'équipe cohérent. Or, force est de constater que le renforcement des contacts avec la clientèle et le développement du travail en équipe ont entraîné une revalorisation des compétences personnelles et sociales au détriment quelquefois des compétences opérationnelles ou professionnelles.

Se pose alors, pour des pays touristiques en développement, la question de la contribution de la formation au développement du tourisme!

Il semble évident que, dans un contexte général de remise à niveau de l'économie, l'industrie touristique est appelée à jouer un rôle primordial. Le Maroc s'empresse aujourd'hui de mettre en place un certain nombre de mesures, notamment en matière d'éducation et de formation, afin de relever les défis de la mondialisation. La principale action vise l'amélioration et le développement du système de formation pour la satisfaction des besoins en compétences des entreprises, la promotion de l'emploi des jeunes et l'amélioration de l'employabilité des travailleurs du secteur. Une autre réforme, aussi importante que la première, est engagée à la fin de l'année 1999 pour la réalisation d'une "Charte nationale d'éducation et de formation ", afin de réaliser l'adéquation tant attendue entre l'éducation et son environnement économique (Ministère du Développement social, 2000).

\section{De la problématique générale de la formation professionnelle au Maroc}

La technologie mondiale progresse rapidement et impose à l'individu des rythmes d'acquisition de nouveaux savoirs souvent complexes et abstraits en termes d'assimilation et d'application, dans les domaines de spécialisation. Cette exigence de compétences constamment renouvelées pousse les pays en développement où le niveau d'instruction générale est encore modeste à des révisions coûteuses des programmes et des orientations. Les décideurs et les formateurs sont conscients des requêtes de l'heure. Mais, comment gérer, dans cette industrie sensible et variable, le décalage grandissant entre les compétences anciennement acquises et celles nouvellement requises. Et l'on comprend que l'évolution mondiale du tourisme influence considérablement l'orientation et le choix de contenu des programmes de formation.

Les changements assez rapides font que les compétences des diplômés en tourisme ne semblent pas toujours répondre de façon appropriée aux besoins réels d'une profession jaugée à l'aune des pays développés. Celle-ci est régulièrement confrontée à une évolution technologique qui bouleverse les pratiques et les métiers, y compris dans les pays avancés. Mais ces derniers ont les moyens matériels, scientifiques et humains pour y faire face.

II existe à ce titre une littérature spécifique qui traite du phénomène appelé « obsolescence professionnelle ». Joannaert et Vander Borght (1999: 10-55) se sont intéressés à ce concept qui définit l'obsolescence comme la difficulté d'un individu à renouveler sa motivation afin d'acquérir constamment de nouvelles connaissances, habitudes et aptitudes. Quant à Caillot et Raisky (1996: 94-117), ils parlent du degré qui ferait défaut et empêcherait l'individu de s'actualiser ou de se renouveler quant aux nouvelles connaissances et autres habiletés nécessaires au maintien d'une performance professionnelle appropriée. En fait,

\section{Tableau 1}

\section{Le secteur touristique au Maroc: état des lieux}

Le tourisme marocain commence à sortir de sa léthargie au moment où l'industrie traverse, à l'échelle mondiale, des tourbillons. II a enregistré 4,6 millions d'arrivées en 2002, contre 2,5 millions en moyenne de 1990 à 2000. En drainant près de 2,5 milliards de dollars de recettes, soit l'équivalent de 24,7 milliards de dirhams, le tourisme constitue la deuxième source de devises du pays; il contribue à hauteur de $6,2 \%$ à la formation du $\mathrm{PIB}^{1}$ (OMT, 2001). Par ailleurs, on estime à 640000 le nombre d'emplois directs et indirects créés par ce secteur au Maroc, soit 5,8 \% de la population active occupée en 2000 (Ministère du Développement social, 2001).

\begin{tabular}{|c|c|c|c|c|c|c|c|c|c|c|}
\hline \multicolumn{11}{|c|}{ Répartition des offres d'emplois directs et indirects (en milliers) } \\
\hline Indicateurs de l'emploi & 2002 & 2003 & 2004 & 2005 & 2006 & 2007 & 2008 & 2009 & 2010 & cumul \\
\hline $\begin{array}{l}\text { Emplois directs: } \\
1 \text { emploi/chambre }\end{array}$ & 6 & 6 & 6 & 8 & 8 & 1 & 1 & 10 & 10 & 72 \\
\hline $\begin{array}{l}\text { Emplois indirects: } \\
7,5 \text { emplois/chambre }\end{array}$ & 45 & 45 & 45 & 60 & 60 & 60 & 75 & 75 & 75 & 540 \\
\hline Total & 51 & 51 & 51 & 68 & 68 & 68 & 85 & 85 & 85 & 612 \\
\hline Cumul des emplois du secteur & 651 & 702 & 753 & 821 & 889 & 957 & 1042 & 1127 & 1212 & - \\
\hline Impact sur la population & 3255 & 3510 & 3765 & 4105 & 4445 & 4785 & 5210 & 5635 & 6060 & - \\
\hline
\end{tabular}

Source : Ministère du Développement social, 2001. 
En ces temps de crises mondiales persistantes, la stabilité politique, la proximité de l'Europe et la grande diversité géographique du territoire font partie des avantages gratifiants qui placent le Maroc parmi les premiers pays africains en matière de tourisme, avec $12,58 \%$ du marché touristique continental.

En conséquence, le tourisme a été reconduit dans ses fonctions de moteur de développement et a fait l'objet d'une stratégie à long terme.

Pour accompagner l'élan escompté pour le secteur, l'Office national marocain du Tourisme a prévu un budget de promotion du Maroc à l'étranger qui devait dépasser les 30 millions d'euros en 2004 et pourrait avoisiner 100 millions d'euros au-delà de 2010.

La détermination du gouvernement à assurer le suivi de ses engagements est visible dans la mise en place de conditions optimales qui permettent un développement rapide et soutenu du tourisme, notamment en termes d'infrastructures de base et de création d'un environnement incitatif pour l'investissement.

\section{Les insuffisances du secteur du tourisme}

Pour opérer dans la visibilité les changements nécessaires, les responsables des départements de formation ont étudié et détecté les faiblesses du secteur et ont pris des prédispositions nécessaires pour en définir les actions correctives et de réajustement ainsi que les axes prioritaires. Ces faiblesses se résument comme suit (Accord-Cadre, 2001) :

- baisse de la qualité des prestations au sein des établissements d'accueil,

- insuffisance des infrastructures de loisirs et d'animation,

- vétusté d'une partie du parc hôtelier national,

- absence d'investissements dans l'entretien et la maintenance,

- inadéquation entre la formation continue et les besoins du secteur.

À vrai dire, les professionnels marocains du tourisme n'ont de cesse de lancer des signaux d'alarme pour sensibiliser les responsables quant à la pénurie d'une main-d'œuvre spécialisée et qualifiée. Ces alertes périodiques ont abouti à la mise en place de sérieuses réformes visant plusieurs aspects de la formation professionnelle et technique (programmes d'études, formation des enseignants et des conseillers pédagogiques...).

Pour accéder aux savoirs pratiques, des partenariats entre l'entreprise et le système de la formation sont envisagés. Le développement quantitatif du mode de la formation par apprentissage, malgré les résultats mitigés obtenus avec la formation par alternance, est assez important. Ces types de formation ont permis, ces dernières années, de doubler, voire de tripler les effectifs dans les établissements de formation professionnelle.

La volonté de l'État de favoriser la scolarisation des jeunes adolescents de milieux défavorisés qui ont abandonné leurs études relève d'une nouveauté, appuyée par des expertises et des coopérations internationales.

\section{Le dispositif de formation professionnelle existant}

Au Maroc, on compte actuellement un peu plus de 50 établissements qui œuvrent dans le domaine de la formation professionnelle touristique et hôtelière. Trois opérateurs de formation alimentent régulièrement le marché du travail en lauréats qualifiés :

- le département du Tourisme compte 11 instituts et 4 centres de qualification professionnelle ;

- l'office de la Formation professionnelle et de la Promotion du Travail gère 3 instituts sectoriels, 7 centres de qualification offrant des formations cuisine/restauration et un centre d'accueil ;
- le secteur de la formation professionnelle privée (Ministère du Développement social, 2001) s'occupe des écoles qui offrent des formations en tourisme et hôtellerie (8 à Agadir et 6 à Casablanca).

En 2003-2004, les effectifs des trois opérateurs de formation totalisent plus de 8000 stagiaires toutes filières et tous modes de formation confondus.

Toutefois, la multiplication des filières souffre d'un manque de coordination entre elles et de correspondance entre les filières et les diplômes. Cela entraîne une incidence quelque peu néfaste sur les coûts de formation et complique le travail des entreprises au moment du recrutement.

Le développement de la formation par apprentissage, à caractère quantitatif, a montré ses limites alors que celui de la formation par alternance demeure mitigé (projet MEDA II). Toutefois, sur le plan quantitatif, ces modes de formation aussi ont permis, ces dernières années, de doubler, voire de tripler les effectifs dans les établissements de formation professionnelle malgré une insuffisance identifiée d'équipements d'accompagnement.

L'État et la profession ont décidé conjointement de "planifier, sur la décennie, les rythmes de formation à adopter pour satisfaire les besoins du secteur et de définir en conséquence les différents programmes d'action pour orienter vers les métiers du tourisme un nombre suffisant de diplômés et de planifier le programme d'insertion professionnelle de ces lauréats par le secteur privé » (Ministère du Développement social, 2001).

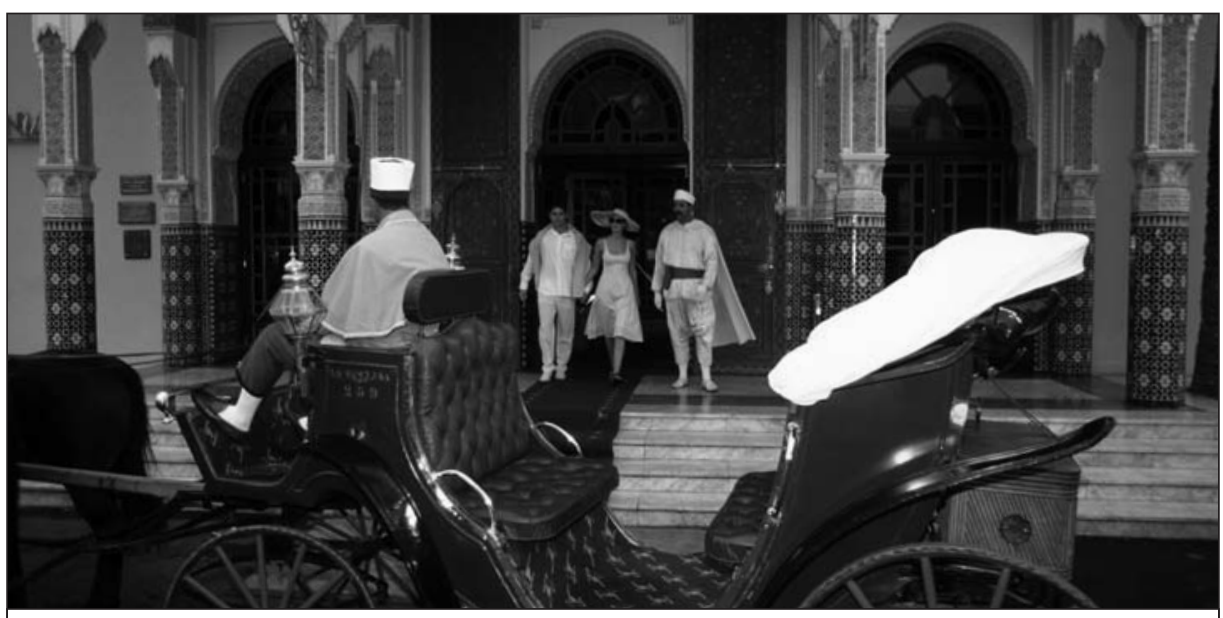

Hôtel la Mamounia dans la ville de Marrakech (Maroc).

Photo : tourisme-marocain.com 


\section{L'offre d'emploi à l'horizon 2010}

Le Maroc espère faire de la promotion du tourisme une occasion de résorption du chômage, surtout celui des diplômés. Un modèle global de simulation établi à partir des résultats d'une étude sectorielle sur l'adéquation «formation - emploi ", réalisée par le Bureau international du travail (BIT) en se basant sur les normes de l'Organisation mondiale du tourisme, a permis de (Ministère du Développement social, 2001) :

- cerner année par année l'évolution de l'offre d'emplois au cours de la prochaine décennie,

- définir le besoin d'emplois par filière,

- estimer le besoin d'emplois par niveau de qualification.

Les résultats de l'étude ont démontré qu'à I'horizon 2010 la capacité d'emplois directs sera de l'ordre de 72000 offres et celle des emplois indirects d'environ 540000 , soit 612000 nouveaux emplois au total. Au cours de cette période, le besoin d'emploi évoluera selon trois paliers de:

- 6000 emplois par an, de 2002 à 2004,

- 8000 emplois par an, de 2005 à 2007,

- 10000 emplois par an, de 2008 à 2010.

Ainsi, $72 \%$ des besoins d'emplois se déclineront comme suit :

- techniciens supérieurs (7\%),

- techniciens moyens (25\%),

- agents qualifiés (40\%).

Le département de la Formation professionnelle, en étroite collaboration avec les opérateurs de formation, a élaboré un plan de développement intégré de la formation professionnelle dans le secteur du tourisme pour juguler certaines crises et pouvoir faire face aux imprévus.

\section{Stratégie de développement de la formation professionnelle dans le secteur du tourisme}

Les actions privilégiées dans le cadre du plan de développement de la formation professionnelle reposent sur (Ministère du Développement social, 2001) :

- l'extension du dispositif de formation,

- la consolidation du dispositif de formation existant,

- la mise en place et le développement d'autres modes de formation.
Les priorités vont à la formation continue, à la formation par apprentissage et à la formation qualifiante jugées nécessaires au décollage du tourisme. La réalisation de ce plan de développement composé de cinq principaux axes doit pouvoir marier réalisme et empirisme en agissant sur :

- La consolidation de l'existant par une mise à niveau progressive des ressources humaines (formation, certification des formateurs, perfectionnement du personnel technico-pédagogique...).

- La dynamisation de la formation continue par la création d'un Groupement interprofessionnel d'aide au conseil, ayant pour but la promotion de la formation continue et la réalisation d'un programme sectoriel d'alphabétisation fonctionnelle au profit des salariés des entreprises du secteur. Ce programme sera financé dans le cadre du système des contrats spéciaux de formation.

- Le développement d'une formation professionnelle par apprentissage au profit des 18000 apprentis prévus à l'horizon 2010.

- La mise en place d'une formation qualifiante au profit des filières du tourisme, destinée aux diplômés chômeurs de l'enseignement supérieur (1000 diplômés à I'horizon 2010).

- L'extension du dispositif de formation publique existant, soit 13 établissements existants et 8 autres à créer, pour permettre une capacité d'accueil additionnelle de 3716 places pédagogiques, portant ainsi la capacité d'accueil globale

Tableau 2

Besoins d'emplois par filière professionnelle

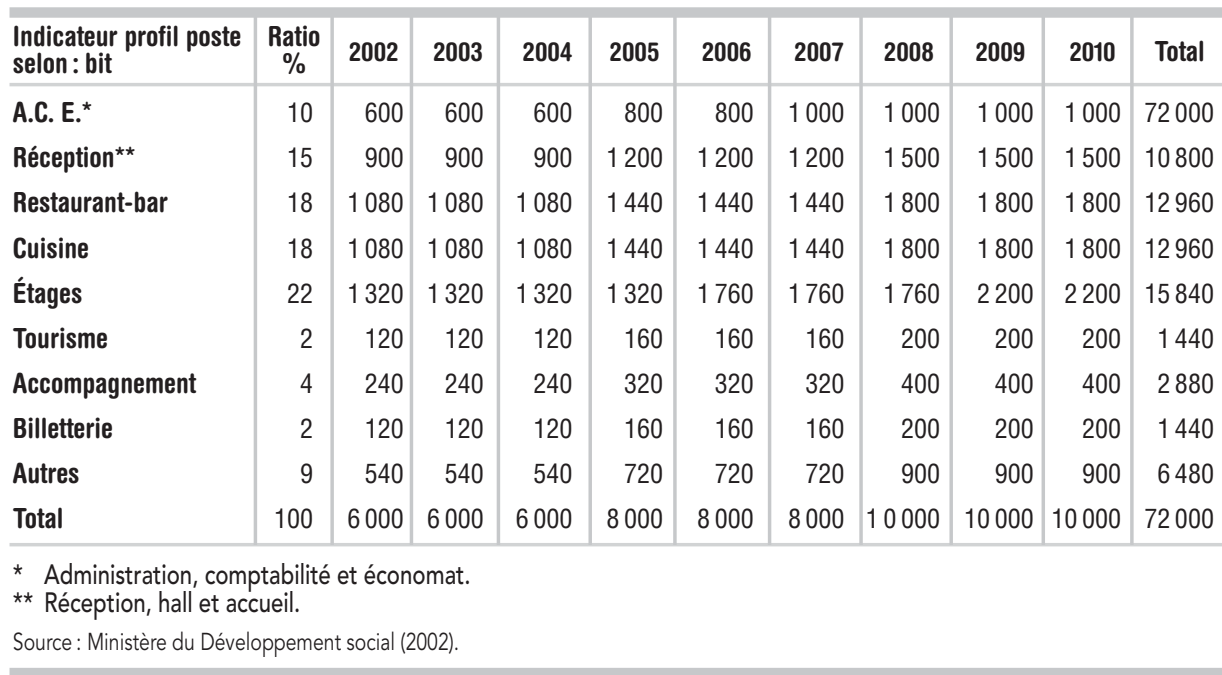

à 7915 places à I'horizon 2010. S'y ajoute la part de la formation professionnelle privée, avec 2400 places pédagogiques nouvelles, portant ainsi la capacité d'accueil à 3565 places à l'horizon 2010. Le cumul des lauréats sur la période 2002-2010 atteindrait 52140 lauréats.

Enfin le risque de se retrouver avec des lauréats formés sans possibilité d'insertion n'est pas à exclure. Mais tout dépendra du comportement du secteur pour les dix ans à venir.

II serait souhaitable alors que les acteurs des différents projets travaillent en collaboration et se concertent régulièrement à partir de lignes directrices afin de guider le développement en cours et à venir et de doter le Maroc d'un plan cohérent de développement de la formation en tourisme et hôtellerie.

\section{Bilan}

Dans le contexte touristique actuel du Maroc, l'importance de l'éducation n'est plus à démontrer. La gestion de l'offre de formation constitue un enjeu central, étant donné la priorité accordée au tourisme et au regard aussi de la montée du chômage. À cet effet, il va falloir se questionner sur la possibilité réelle de l'État à pouvoir gérer l'offre de formation de manière à répondre convenablement aux besoins de la profession et aux pressions de la démographie.

La réponse la plus pertinente est, semble-t-il, d'assouplir la planification en intégrant l'entre- 
prise, en tant qu'acteur de la formation, soit de manière directe par la formation en alternance ou en apprentissage, soit à travers son engagement continu dans l'élaboration des cartes et des programmes de formation. L'État aura grand peine à être à la fois juge et partie!

Par ailleurs, on peut s'interroger, légitimement, sur la capacité des entreprises, ne serait-ce qu'au regard des mauvaises habitudes prises dans la gestion de ressources humaines, à prévoir et à promouvoir leurs besoins en main-d'œuvre et en qualification (Projet Medall).

On le voit bien, les pratiques de gestion des ressources humaines suscitent encore quelques interrogations dans le secteur touristique. C'est pour cette raison qu'en ce qui touche le schéma de rapprochement entre l'offre de formation et les exigences du secteur, les entreprises semblent d'ores et déjà acculées à manifester leur volonté et à prouver leur capacité à contribuer efficacement à la gestion concertée de l'offre de formation.

Cette prédisposition à s'ouvrir sur leur environnement professionnel dépend, à n'en pas douter, de l'existence et de l'efficacité d'une organisation des entreprises en groupements par secteur, par branche ou par fédération... Elles n'ont d'autres choix que d'afficher une réelle volonté de participation au développement de la formation professionnelle en appuyant l'information et la sensibilisation des entreprises sur la nécessité de gérer l'emploi et les carrières de manière à favoriser le dialogue avec tous les acteurs de ce domaine (professionnels, autorités, travailleurs, formateurs...).

De ce fait, le rôle de l'État est de veiller à l'exécution de la politique de formation, car la participation des entreprises ne constitue pas en elle-même une garantie de participation. II doit en évaluer l'efficacité, à travers le suivi de l'insertion professionnelle des diplômés, notamment par la mise en place d'un observatoire du tourisme et une connaissance réelle du marché du travail.

Le rapprochement du monde de l'entreprise à celui de la formation nécessite, en plus des actions communes, un langage commun. La notion de compétences depuis quelques années constitue le moyen d'expression des besoins des entreprises dans la désignation officielle des fonctions et de leur niveau au sein des sociétés touristiques par le biais des classifications. Une offre de formation optimale, c'est-à-dire celle qui prétend accroître son efficacité, requiert d'être à l'écoute de la demande des entreprises et, de ce fait, nécessite une intervention sur plusieurs fronts : révision continuelle des programmes, assistance des pouvoirs publics, innovation dans les méthodes d'enseignement, développement de la formation continue, recyclage des formateurs et développement d'associations professionnelles).

Le besoin de développer les ressources humaines en quantité et en qualité dans le secteur semble indispensable pour accompagner et réussir une entreprise de soutien de la demande à partir des nouvelles aspirations des consommateurs. Répondre à ces défis, c'est créer de vrais emplois durables et «professionnalisables » en répondant également aux besoins sociaux; c'est faire preuve d'innovation ; c'est mobiliser les ressources humaines à la hauteur des enjeux et des espoirs...

Les contrats spéciaux de formation ont pour but d'amener les entreprises à intégrer la formation dans leur plan de développement en leur accordant une assistance financière pour élaborer et exécuter leur plan de formation. Les contrats spéciaux de formation sont régis par un manuel des procédures qui définit la nature des actions finançables par ce système, les contenus des demandes de financement et les critères de sélection. Sont admissibles au financement des contrats spéciaux de formation toutes les entreprises assujetties à la taxe de formation professionnelle et en situation régulière vis-à-vis de la Caisse nationale de Sécurité sociale.

Fatima Zohra Guertaoui est doctorante à I'Université Cadi Ayyad où elle collabore aux travaux de recherche de l'École doctorale internationale de tourisme (EDIT). Elle enseigne la gestion hôtelière à l'Institut supérieur d'industrie hôtelière et de restauration de Marrakech.

\section{Notes}

1 Les recettes du tourisme arrivent juste après les sommes rapatriées par les MRE, marocains Résidants à l'Étranger.

\section{Bibliographie}

Aberlen, Jacques, Jean Viard, et Richard Lewy (rapporteurs) (1997), Étude prospective des gisements d'emplois générés par la demande touristique, Paris, Conseil national du tourisme, 93p.

Abdellaoui, Bouchaib (1999), «Structure de l'emploi et besoins de qualification dans l'hôtellerie marocaine ", dans Mohamed Berriane et
Hebert Popp (dir.), Le tourisme au Maghreb : diversification du produit de développement régional et local, Rabat, Actes du $5^{\mathrm{e}}$ colloque maroco-allemand de Tanger, p. 95-10.

AFPA (2003), Appui au développement de la formation professionnelle dans les secteurs du tourisme, du textile et des NTIC, Projet MEDA II, Étude d'opportunité et de faisabilité pour la création de 8 établissements de formation professionnelle dans le secteur du tourisme, Fiche synthétique, Rapport intermédiaire, octobre, 71 p.

Belkheiri, O. (1999), "L'emploi et la formation dans le secteur touristique au Maroc, pour une approche dynamique des rapports entre le système productif et le système de formation ", thèse de doctorat, Université de Montpellier I.

Cornu, Roger (2001), Éducation, savoir et production, Bruxelles, Édition de l'Université de Bruxelles.

Clanet, Claude, et Benoît Jeunier (2000), Approches systémiques et recherches en sciences de l'éducation, Toulouse, Presses universitaires du Mirail.

Donnay, Jean, et Marc Bru (2002), Recherches, pratiques et savoirs en éducation, Bruxelles, De Boeck.

Franco, Arlette (dir.) (1999), Rapport sur les diplômes et les formations aux métiers du tourisme, Paris, Assemblée Nationale, 90 p., [http:// www.ladocumentationfrancaise.fr/brp/notices/ 034000418.shtml].

Guertaoui, Fatima Zohra (2001), Développement de la formation continue dans le secteur hôtelier au Maroc : Résultats et conclusions d'une enquête menée auprès des établissements hôteliers classés de Marrakech, mémoire de fin d'études du DESS, Marrakech, Faculté des lettres.

Joannert, Philippe, et Cécile Vander Borght (1999), Créer des conditions d'apprentissage: un cadre de référence socioconstructiviste pour une formation didactique des enseignants. Perspective en éducation, Bruxelles, De Boeck Université.

Ministère de l'Économie, des Finances et du Tourisme et de la Privatisation et la CGEM (2001), Accord-cadre 2001-2010, Marrakech.

Ministère du Développement social, de la Solidarité, de l'Emploi et de la Formation professionnelle (2000), Élaboration des référentiels d'emploi, des compétences et des cahiers des charges de formation dans le secteur tourisme/ hôtellerie et restauration. Phase I: Portrait du secteur de formation tourisme-hotellerie-restauration, Groupement CIDE (Québec) - CENTAURE (Maroc), mai, 121 pages + annexes.

Ministère du Développement social, de la Solidarité, de l'Emploi et de la Formation professionnelle (2001), Tourisme, Hôtellerie et Restauration. Référentiels de formation, Groupement CIDE (Québec) - CENTAURE (Maroc), juin, non paginé.

Organisation mondiale du tourisme (2001), Tourism Market Trends - Africa 2001, [www.world-tou rism.org], Édition Madrid Espagne.

Py, Pierre (1992), « Le tourisme phénomène économique ", La documentation française.

Raisky, Claude, et Michel Caillot (dir.) (1996), Au-delà des didactiques, le didactique. Débats autour de concepts fédérateurs, Bruxelles, De Boeck.

UNESCO, [http://unescostat.unesco.ord/database/]. 